\title{
Octave-spanning lithium niobate soliton microcombs
}

\author{
Yang $\mathrm{He}^{1}{ }^{1}$ Raymond Lopez-Rios ${ }^{2}$, Qifan Yang ${ }^{3}$, Jingwei Ling ${ }^{1}$, Mingxiao Li ${ }^{1}$, Kerry \\ Vahala $^{3}$, and Qiang Lin ${ }^{1,2}$ \\ ${ }^{1}$ Department of Electrical and Computer Engineering, University of Rochester, Rochester, New York 14627 \\ ${ }^{2}$ Institute of Optics, University of Rochester, Rochester, New York 14627 \\ ${ }^{3}$ T. J. Watson Laboratory of Applied Physics, California Institute of Technology, Pasadena, California 91125
}

\begin{abstract}
We report lithium niobate soliton microcombs with spectral bandwidths ex-ceeding one octave and spanning $125 \mathrm{THz}$ to $268 \mathrm{THz}$. ( 2021 The Author(s)
\end{abstract}

\section{Main Text}

Kerr soliton microcombs provide a path towards chip-scale miniaturization of frequency comb systems with applications spanning frequency metrology and precision time keeping to optical communications [1]. However, most applications will require an octave-spanning comb spectrum so as to implement the $f$-to- $2 f$ self-referencing technique [2]. And, so far this spectral width has only been possible in silicon nitride microresonators [3-5]. Here, we report octave-spanning soliton microcombs produced in monolithic lithium niobate (LN) microresonators. Dispersion-engineered dispersive wave generation from single and multi-soliton states is applied to generate comb spectra up to 1.15 octave.
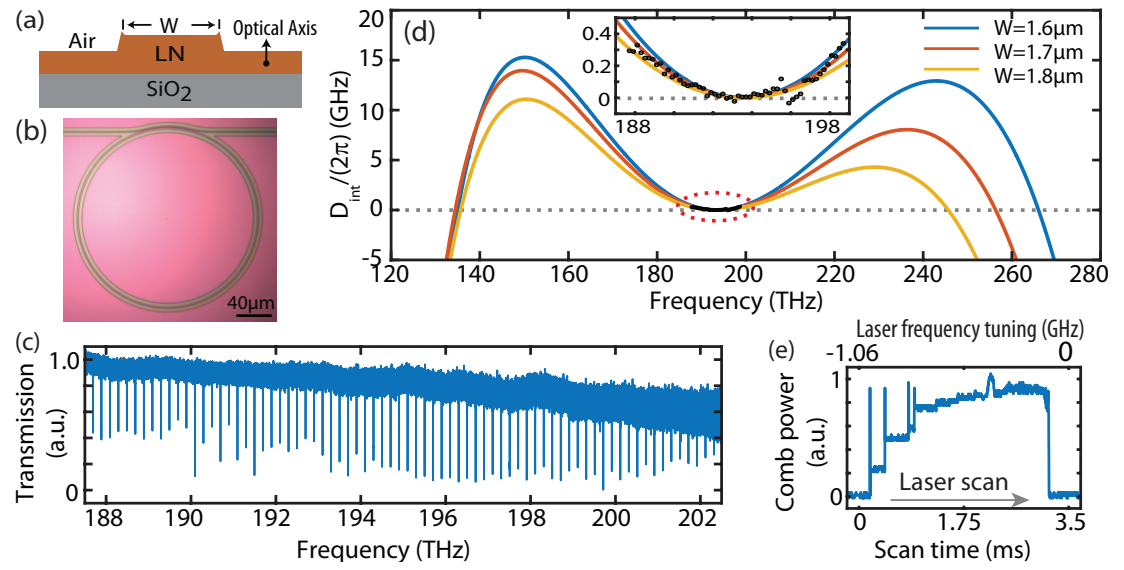

Fig. 1. (a) Schematic of the LN waveguide cross section. (b) Optical micrograph of a fabricated LN microring. (c) Frequency scan of microresonator transmission spectrum (waveguide width of $1.6 \mu \mathrm{m})$. (d) Simulated LN waveguide integrated dispersion $D_{\text {int }} / 2 \pi\left(D_{\text {int }}=\frac{1}{2} \mu^{2} D_{2}+\right.$ $\left.\frac{1}{6} \mu^{3} D_{3}+\cdots\right)$. The inset provides a spectral zoom-in region indicated by the dotted oval in the main panel. Experimental dispersion data are shown as black dots and give $D_{2} /(2 \pi)=0.84 \mathrm{MHz}$. (e) Pump laser frequency scan of comb power showing the soliton steps.

The LN microring resonators were fabricated on a $600 \mathrm{~nm}$-thick $\mathrm{z}$-cut $\mathrm{LN}$-on-insulator wafer, with a radius of $100 \mu \mathrm{m}$ (free spectral range of $199.7 \mathrm{GHz}$ ) and an etching depth of about $300 \mathrm{~nm}$ (Fig. 1(a,b)). The device features a loaded optical Q of 1.15 million in the telecom band with good waveguide coupling (Fig. 1(c)). Groupvelocity dispersion (GVD) was engineered by varying the waveguide width as shown in Fig. 1(d) where finite element method simulations of waveguide widths from 1.8 to $1.6 \mu \mathrm{m}$ are shown. Over this width range, the high-frequency phase-matching point for dispersive wave (DW) generation shifts from 245 to $266 \mathrm{THz}$, while the low-frequency point around $135 \mathrm{THz}$ is nearly unaffected. At the same time, a spectral region of anomalous GVD in the telecom band is available for soliton formation. The measured dispersion profile in the telecom band (shown in the inset of Fig. 1(d)) agrees well with the numeric simulations. A pump wave was coupled into a cavity mode around $193 \mathrm{THz}$, and soliton states were observed to self start [6]. Soliton steps are shown in Fig. 1(e).

Figure 2(a)-(c) show the optical spectra of the single-soliton states produced in LN microresonators having different waveguide widths. In general, the soliton microcombs exhibit broadband spectra extending from below 
$140 \mathrm{THz}$ to above $240 \mathrm{THz}$. The high-frequency DW is located around $238 \mathrm{THz}$ in the $1.8-\mu \mathrm{m}$ resonator. It is observed to shift to $\sim 255 \mathrm{THz}$ in the $1.7-\mu \mathrm{m}$ resonator, and then to shift further to $\sim 264 \mathrm{THz}$ in the $1.6-\mu \mathrm{m}$ resonator. On the other hand, the spectral bump located around $140 \mathrm{THz}$ is likely due to the low-frequency DW. These observations agree well with the simulated dispersion profiles in Fig. 1(b). In particular, the single-soliton state produced in the 1.6- $\mu \mathrm{m}$ resonator (Fig. 2(c)) exhibits an octave-spanning spectrum from $128 \mathrm{THz}$ to $267 \mathrm{THz}$. Interestingly, the extremely broad bandwidth leads to strong intrapulse Raman scattering, with a prominent soliton self-frequency shift (SSFS) of $5.4 \mathrm{THz}$. The octave-spanning spectrum is clearer in a multi-soliton state (Fig. 2(d)) where the comb line powers are enhanced. The spectrum spans $125 \mathrm{THz}$ to $268 \mathrm{THz}$. With the enhanced comb line powers, the comb modes become more visible in the spectral region around 230-260 THz. The small comb amplitudes in this region are primarily due to the un-optimized external coupling of resonator to the bus waveguide. The major features observed in the octave-spanning soliton combs, such as spectral extent of combs, magnitude of SSFS, spectral locations of the DWs, and the smaller comb amplitudes around 230-260 THz, are reproduced in numerical simulations shown in Fig. 2(e) and (f).

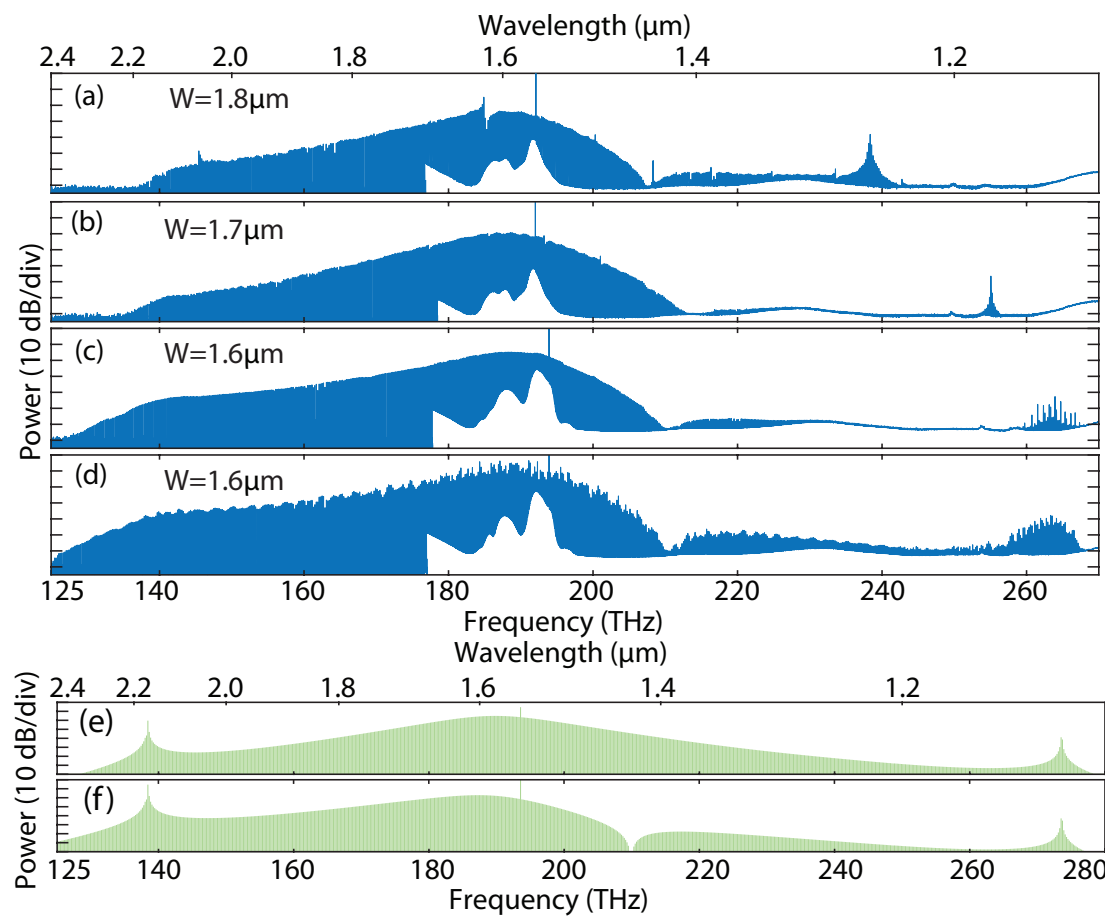

Fig. 2. Optical spectra of soliton microcombs produced in LN microresonators with different waveguide widths. (a)-(c) are single-soliton states, and (d) is a multi-soltion state. The on-chip pump power is $\sim 396$ and $524 \mathrm{~mW}$ for (a) and (b), respectively; and $\sim 600 \mathrm{~mW}$ for (c) and (d). (e) and (f) are numerically simulated optical spectra of internal (e) and transmitted (f) soliton combs (1.6- $\mu \mathrm{m}$ resonator) using a generalized Lugiato-Lefever equation.

In summary, we demonstrate octave-span lithium niobate soliton microcombs, which are a critical step for realizing intergrated $f$-to- $2 f$ self-referenced microcombs on a single LN chip.

This work was supported by Defense Threat Reduction Agency (HDTRA11810047).

\section{References}

1. T. J. Kippenberg, et al, ”Dissipative kerr solitons in optical microresonators," Science 361 (2018).

2. S. A. Diddams, K. Vahala, and T. Udem, "Optical frequency combs: Coherently uniting the electromagnetic spectrum," Science 369 (2020).

3. D. T. Spencer, et al, "An optical-frequency synthesizer using integrated photonics," Nature 557, 81 (2018).

4. Q. Li, et al, "Stably accessing octave-spanning microresonator frequency combs in the soliton regime," Optica 4, 193 (2017).

5. M. H. Pfeiffer, et al, "Octave-spanning dissipative kerr soliton frequency combs in $\mathrm{Si}_{3} \mathrm{~N}_{4}$ microresonators," Optica 4, 684-691 (2017).

6. Y. He, et al, "Self-starting bi-chromatic $\mathrm{LiNbO}_{3}$ soliton microcomb”, Optica, 6, 1138-1144 (2019). 